\title{
Producción y valor proteico de Pleurotus ostreatus en la región sur de Ecuador
}

\author{
Darío Cruz ${ }^{1 *}$, Daniel Capa ${ }^{2}$, Diego Maza ${ }^{3}$, Ruby 0jeda², Ángel Benitez ${ }^{1}$ \\ 'Museo de Colecciones Biológicas, Departamento de Ciencias Biológicas, Grupos de Investigación \\ MS2E y BIETROP; Universidad Técnica Particular de Loja, San Cayetano Alto s/n C.P. 1101 608, Loja, \\ Ecuador. \\ 2 Departamento de Ciencias Biológicas, Grupo de Investigación BIETROP; Carrera de Ingeniería \\ Agropecuaria; Universidad Técnica Particular de Loja, San Cayetano Alto s/n C.P. 1101 608, Loja, \\ Ecuador. \\ ${ }^{3}$ Departamento de Química y Ciencias Exactas, Grupo de Investigación GIMA; Universidad Técnica \\ Particular de Loja, San Cayetano Alto s/n C.P. 1101 608, Loja, Ecuador. \\ *Autor para correspondencia / Corrersponding author: djcruz@utpl.edu.ec
}

\section{Production and protein value of Pleurotus ostreatus in the southern region of Ecuador}

\begin{abstract}
Mushrooms feed on organic matter, many of them with high nutritional value and poorly used. Large-scale crops require a strong economic investment to control temperature and humidity variables. The overall aim of this study is to cultivate Pleurotus ostreatus by hand without further automated control of temperature and humidity, optimizing the use of agro-industrial residues: rice husk (Oriza sativa L.), coffee husk (Coffea arabica L.) and sawdust (sawn wood particles), as substrates in the production of this species. Determining production rate (TP), biological efficiency (EB), and the percentage of protein. The results indicate that the combination of substrates, rice husk + coffee husk + sawdust (T1) was better in production rate $(80,20 \%)$ and biological efficiency $(15,23 \%)$ over only a sawdust substrate (T2). The percentage of protein 40\% (T2) and 35\% (T1) were not significantly different. The cultivation of P. ostreatus is shown as a sustainable alternative source of protein in the diet of vulnerable populations.
\end{abstract}

Keywords: sawdust, rice husk, coffee husk, protein.

Editado por /

Edited by:

Eva O.L. Lantsoght

Recibido /

Received:

01/06/2020

Aceptado /

Accepted:

07/10/2020

Publicado en línea

/ Published online:

$15 / 12 / 2020$

\section{Resumen}

Los hongos se alimentan de materia orgánica, muchos de ellos con alto valor nutricional y pobremente aprovechados. Cultivos a gran escala requieren inversiones económicas fuertes para controlar variables ambientales. Este estudio buscó cultivar Pleurotus ostreatus artesanalmente sin mayor control automatizado de temperatura y humedad, optimizando el uso de residuos agroindustriales: cascarilla de arroz (Oriza sativa L.), cascarilla de café (Coffea arabica L.) y aserrín (partículas de la madera aserrada), como sustratos en la producción de esta especie. Se determinó la tasa de producción (TP), eficiencia biológica (EB), y el porcentaje de proteína. Los resultados indicaron que la combinación de sustratos, cascarilla de arroz + cascarilla de café + 
aserrín (T1) fue mejor en tasa de producción (80,20\%) y eficiencia biológica $(15,23 \%)$ sobre un sustrato de aserrín (T2). El porcentaje de proteína 40\% (T2) y 35\% (T1) no tuvieron diferencia significativa. El cultivo de P. ostreatus se muestra como una fuente alternativa sustentable de proteína en la alimentación de poblaciones vulnerables.

Palabras clave: aserrín, cascarilla de arroz, cascarilla de café, proteína.

\section{INTRODUCCIÓN}

El basidiomiceto Pleurotus ostreatus (Jacq.) P. Kumm; pertenece al orden Agaricales y familia Pleurotaceae, es muy importante en roles ecológicos como la degradación de materia orgánica, principalmente lignina y compuestos hemicelulíticos [1]. P. ostreatus es comestible y ha sido colectado y consumido desde la antigüedad [2], de fácil cultivo en diversos residuos derivados de la agroindustria, como desperdicios del aserrado de madera (aserrín) [3, 4]. Varios estudios [5, 6], han testeado la productividad y rendimiento de proteína de esta especie en diversos sustratos, por ejemplo, hojas de plátano y aserrín (38,5\% de proteína), paja de trigo (25,6\% de proteína), eucalipto (25,4\% de proteína), y en álamo (22,9\% de proteína). En Ecuador, a nivel de laboratorio se ha evaluado principalmente la eficiencia biológica en bagazo de caña [7, 8], sustratos como el aserrín, bagazo de caña, y afrecho de trigo, han sido evaluados a nivel productivo en una finca en la provincia de Pastaza [9]. Sin embargo, los estudios son limitados en los que se realice una combinación de varios sustratos.

P. ostreatus tiene un alto valor nutricional [10] conteniendo entre 57\% y $61 \%$ de carbohidratos, $26 \%$ de proteína, $11,9 \%$ de fibra y $0,9 \%$ a $1,8 \%$ de grasas con base a su peso seco, también posee vitaminas como la niacina, tiamina (B1), vitamina B12, vitamina $C$ o ácido ascórbico y minerales como potasio, fósforo y calcio [11]. Por estos elementos y compuestos, además de cultivarse libre de compuestos tóxicos como pesticidas, el consumo de esta especie podría ayudar como inmunoregulador y antitumoral, aportando en el mejoramiento de enfermedades relacionadas con neoplasias como el cáncer [12].

La demanda y consumo de macrohongos (setas y hongos) en países Nórdicos como los europeos está culturalmente muy aceptada [13]. En Latinoamérica y especialmente en el Ecuador, el conocimiento y el consumo de macrohongos no es muy popular, sin embargo, varias comunidades indígenas históricamente han venido aprovechando ciertos cuerpos fructíferos como recurso alimenticio [14]. Es así, que el consumo de P. ostreatus, una especie introducida para Ecuador, y otros hongos comestibles nativos podrían constituirse en un aporte nutricional proteínico comparable con otros suplementos alimenticios $[15,16]$, especialmente en poblaciones vulnerables con recursos económicos limitados.

En este sentido, el presente trabajo busca obtener un mejor rendimiento proteico del hongo P. ostreatus cultivado sin mayor control de temperatura y humedad en dos 
diferentes sustratos, aportando localmente información respecto de los beneficios nutricionales que posee esta especie, y así contribuir con varios puntos detallados en el segundo objetivo de desarrollo sostenible (ODS).

\section{METODOLOGÍA}

\section{Diseño y recolección de datos}

El estudio se llevó a cabo en la Finca privada "La Dolorosa" ubicada en el sector Bombuscaro, vía Romerillos $4^{\circ} 04^{\prime} 02.0^{\prime \prime} \mathrm{S} 78^{\circ} 56^{\prime} 48.6^{\prime \prime} \mathrm{W}$, provincia de Zamora Chinchipe.

\section{Tratamientos}

Se realizó la siembra en dos procesos con 30 réplicas en bolsas plásticas de $1 \mathrm{lb}$ para cada uno de ellas. El tratamiento 1 (T1) es una combinación de cascarilla de café, cascarilla de arroz y aserrín en relación 1:1:1, y el Tratamiento 2 (T2) con aserrín.

\section{Inóculo, siembra y cosecha}

La cepa HUTPL-329 de Pleurotus ostreatus fue reactivada en medio PDA (medio agar papa dextrosa) inóculo primario e incubación a $27^{\circ} \mathrm{C}$. Posteriormente, semillas de trigo con cáscara (aprox. 400gr por frasco) fueron sumergidas en agua entre 12-14 horas. Luego, se retiró el exceso de agua hasta alcanzar aproximadamente un 65 a $75 \%$ de humedad $[17,18]$.

Los diferentes frascos con semillas de trigo fueron esterilizados por autoclave $\left(121^{\circ} \mathrm{C}\right.$ por 15 min a 1,5lb de presión) y fueron inoculados con segmentos de $1 \mathrm{~mm} 2$ (inóculo primario) de P. ostreatus, seguido de incubación a $27^{\circ} \mathrm{C}$ constituyendo así el inóculo secundario. Este último se almacenó hasta alcanzar un llenado completo de micelio, lográndose en unos 10 días promedio. Todo el proceso se lo efectuó en Laboratorio Cultivo y Conservación de Microorganismos de la Universidad Técnica Particular de Loja (UTPL).

Previo a la inoculación del sustrato con el inóculo secundario (un frasco con aprox.400g de semillas colonizadas con micelio sobre $1 \mathrm{lb}$ de sustrato T1 y T2), se esterilizó el sustrato por medio de agua hirviendo por unos 30min. Al igual que el inóculo secundario, el sustrato se preparó con un 65 a 75\% de humedad aproximadamente (prueba de la mano = aplastar el sustrato en la mano y que caigan apenas unas gotas de agua) [19], más carbonato de calcio (cal de construcción) en proporción de 1\% con respecto del sustrato [20].

Las fundas de los tratamientos fueron almacenadas en un cuarto con sombra y temperatura ambiente, fluctuantes según el clima en la localidad, y verificando el periodo de tiempo hasta cumplir las diferentes etapas de desarrollo; micelio, primordios y basidiomas. Los cuerpos fructíferos se cosecharon al alcanzar una madurez adecuada, en referencia, principalmente a la coloración (blanco-grisáceo) y textura (carnosa e hidratada). Réplicas de las cepas se almacenaron en la Micoteca del Laboratorio Cultivo y Conservación de Microorganismos y basidiomas secos de Pleurotus ostreatus se almacenaron en el fungario del Herbario HUTPL. 


\section{Análisis de productividad y proteína}

La productividad de P. ostreatus según los tratamientos se determinó por medio de la evaluación de la tasa de producción (TP), la eficiencia biológica (EB) con las fórmulas de cálculo según [21].

$\mathrm{TP}=\frac{\text { Eficiencia biológica }}{\text { Número de días del proceso }}$
$\mathrm{EB}(\%)=\frac{\text { Peso de hongos frescos }}{\text { Peso del sustrato fresco }} * 100$

Por otra parte el análisis de proteína en los basidiomas partiendo de 0,5g de muestra, se determinó por el método de Kjeldahl (AOAC, USEPA, ISO) (Datos S1), el cual permite calcular la cantidad de nitrógeno presente en el alimento, el mismo que consiste básicamente en ebullición de la muestra en ácido sulfúrico concentrado provocando destrucción oxidativa de la materia orgánica y reducirlo a nitrógeno orgánico [22], para finalmente calcularse, el porcentaje de proteína bruta (\%P), y el porcentaje de nitrógeno $(\% \mathrm{~N})$ aplicando los siguientes componentes en la fórmula: usando al volumen de titulación de la muestra $(\mathrm{V})$; volumen de titulación del blanco (Vb); constante de normalización de HCL 0,1N (K) y peso de muestra en gramos (P).

$\% \mathrm{~N}=\frac{(\mathrm{V}-\mathrm{V} b)^{*} 1,401^{*} \mathrm{~K}}{\mathrm{P}}$

$\% P=\% * 6,25$

\section{Análisis de datos}

Los datos obtenidos para tasa de producción, eficiencia biológica y porcentaje de proteína en función de los tratamientos se analizaron mediante una prueba de Wilconxon. La prueba de Shapiro-Wilk fue menor a 0,05, confirmando que los datos no presentaron una distribución normal.

\section{Resultados y Discusión}

El tiempo de desarrollo de Pleurotus ostreatus (Fig. 1), fue variable entre los dos sustratos o tratamientos (T1 y T2) posterior al día uno de inoculación, siendo así: colonización miceliar completa (30 y 38 días respectivamente); primordios (35 y 45 días respectivamente); fructificación (39 y 50 días respectivamente), repercutiendo en alguno de los parámetros analizados como se explica seguidamente. 

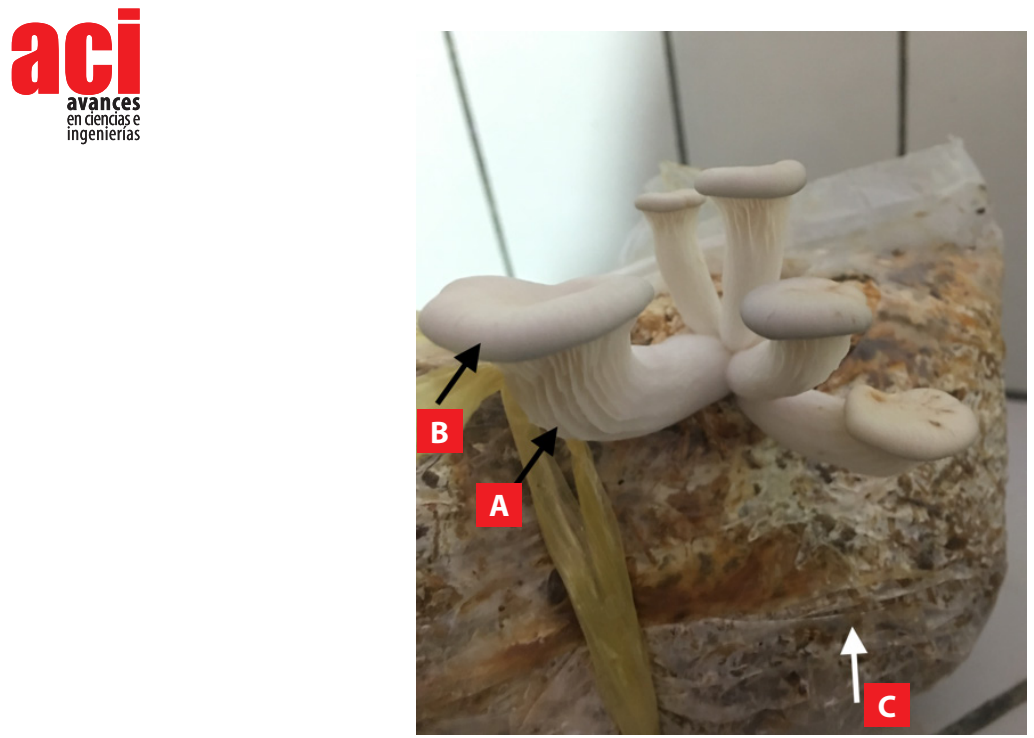

FIGURA 1. Basidiomas de P. ostreatus; lamelas decurrentes (A) y pileo grisáceo (B), hábito cespitoso y/o connado; funda con sustrato (hábitat lignícola), y crecimiento miceliar dicariótico (C).

La tasa de producción (Fig. 2) y la eficacia biológica (Fig. 3) mostraron diferencias significativas entre los tratamientos. Sin embargo, el porcentaje de proteína (Fig. 4) no señaló cambios significativos (Tabla 1).

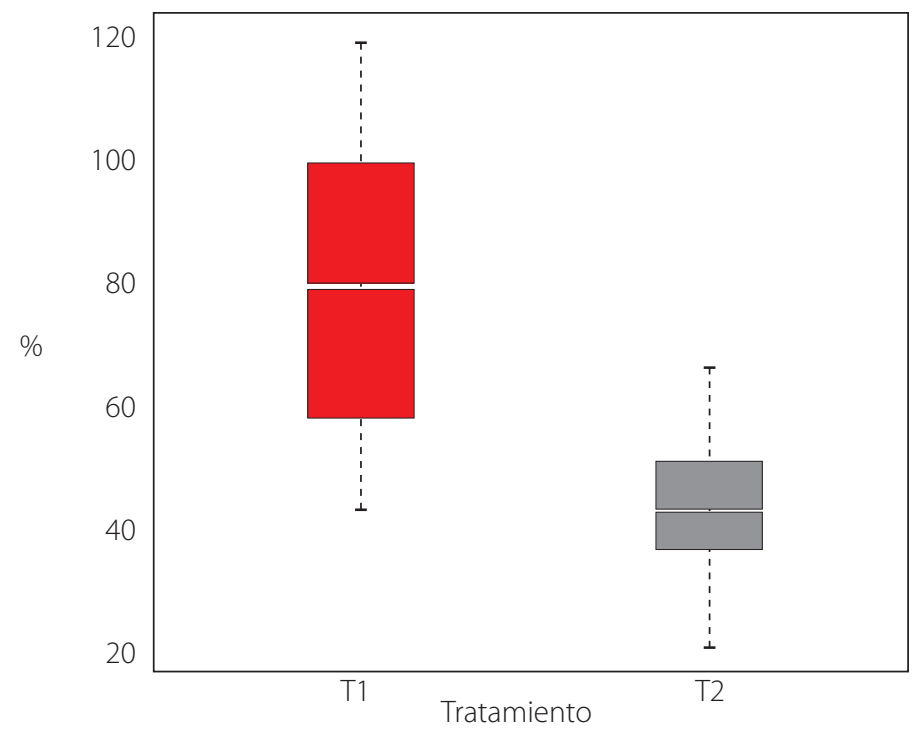

FIGURA 2. Tasa de producción de P. ostreatus entre tratamientos 


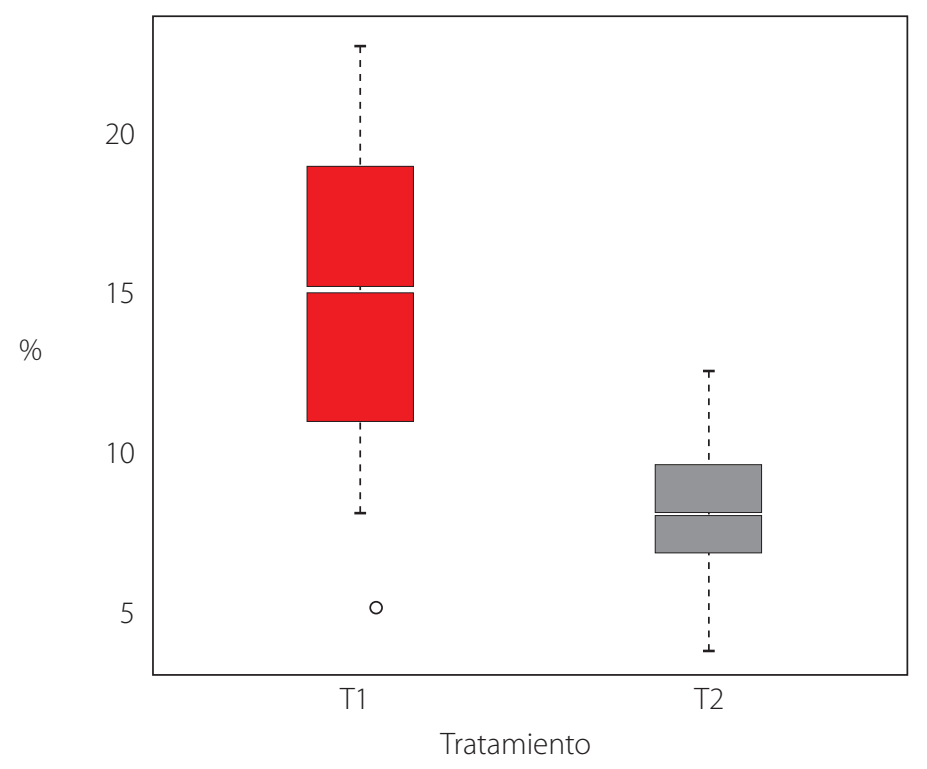

FIGURA 3. Eficiencia biológica de P. ostreatus entre tratamientos.

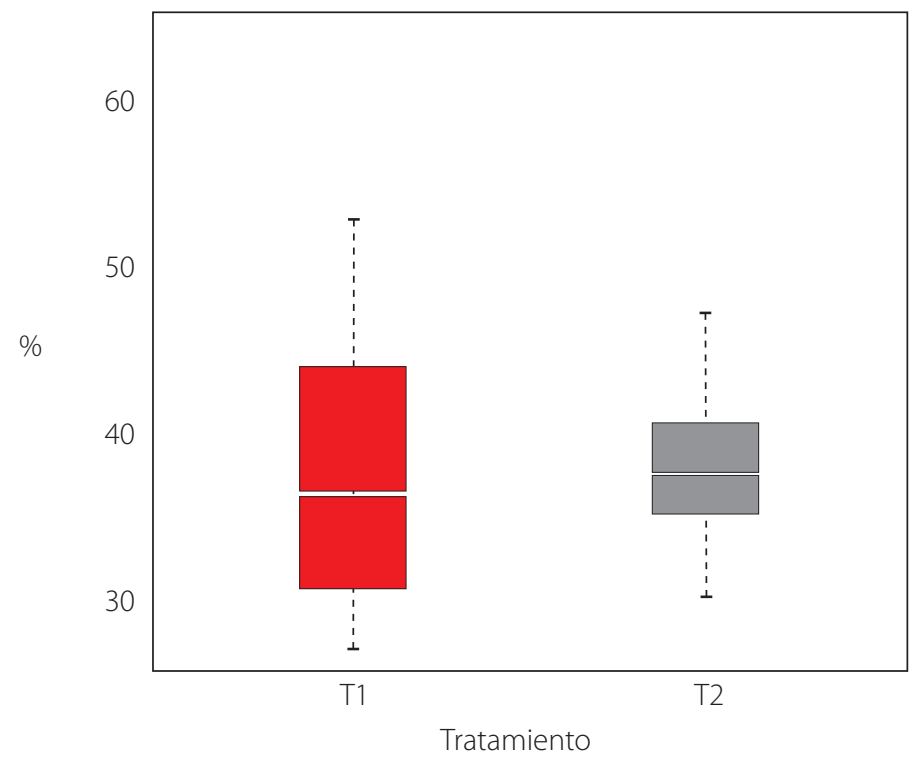

FIGURA 4. Porcentaje de Proteína de P. ostreatus entre tratamientos 
TABLA 1. Datos de significancia de pruebas de supuestos y diferencia de medias entre tratamientos.

\begin{tabular}{|c|c|c|c|c|}
\hline & \multicolumn{2}{|c|}{ Shapiro Wilk } & \multicolumn{2}{c|}{ Wilcoxon Test } \\
\hline Variables & W & P-valor & W & P-valor \\
\hline TP & 0,94622 & 0,01039 & 820 & $4,68 \mathrm{E}-08$ \\
\hline EB & 0,94627 & 0,01044 & 820,5 & $4,48 \mathrm{E}-08$ \\
\hline$\%$ \% & 0,94992 & 0,01543 & 390 & 0,3817 \\
\hline
\end{tabular}

El T1 con una tasa de producción del 80\% TP fue superior al 45\% del T2. La tasa de producción es importante en el cultivo de este macrohongo, encontrándose valores referenciales entre 60 y $80 \%$ aplicando diferentes sustratos [23-25], siendo un indicativo que la combinación de sustratos en el T1 de este estudio es eficiente. No obstante, se debe indicar que los días para observar fructificaciones en el T1 han excediendo aproximadamente en 10 días con respecto de otros estudios [26], lo que puede estar relacionado con cambios de la temperatura.

El T1 con el 15\% de EB fue a superior al T2 con el 8\%. El cual preferencialmente debe ser del 100\% [27-28] garantizando la convergencia y aceptabilidad del sustrato por el hongo. Los valores aquí obtenidos para el T1, con un valor medio del 15\% y un 22,75\% en su valor máximo, son inferiores para valores de EB obtenidos en otros estudios, por ejemplo, se ha reportado entre el 56\% a 160\% de EB para los sustratos de cascarilla de arroz y pulpa de café [23], siguiendo este mismo patrón, otros autores documentaron valores altos de EB para el carrizo con 123,21\% [25]; paja de cebada con 59,95\% [24]; restos de cultivo de frejol y tuza de maíz 64\% y 62\% respectivamente [29]. Así mismo, Caicedo [29] menciona que sustratos como, aserrín, kudzu, hojas de plátano y anturio pueden mantener EB entre $181 \%$ a $257 \%$.

Los bajos porcentajes principalmente en la EB y la variación en tiempo se la atribuyen a la variabilidad de condiciones como temperatura y humedad como se sugiere en [26] provocando estrés de desecación, lo cual disminuye el metabolismo del hongo como se explica en [17]. La temperatura y humedad en la ciudad de Zamora, oscilo entre $25-27{ }^{\circ} \mathrm{C}$ y humedad entre 70 y $95 \%$ respectivamente durante el periodo de siembra y cosecha. Los datos tomados a lo interno del cuarto de cultivo variaron con respecto del ambiente externo obteniendo rangos entre 24 y $26^{\circ} \mathrm{C}$ para temperatura y entre 60-90\% para humedad, permitiendo al menos el desarrollo de los hongos.

Sorpresivamente, los datos de proteína fueron entre 40\% y 35\% para T2 y T1 respectivamente, muy superiores para los reportados para la misma especie entre 1,54\% a 3,10\% (factor de conversión de nitrógeno 4.38) [30], y para la cepa RN2 P. pulmonarius (Fr.) Quél. (sensu auct. P. ostreatus) y la cepa RN82 P. djamor (Ruumph. Ex Fr.) Boedijn con valores de proteína entre 23-43\% [21]. Estos valores de proteína están ligados al tipo de sustrato que se use en el cultivo, por ejemplo, la misma especie de P. djamor cultivado en hojas de plátano y aserrín presenta un porcentaje 38,5 de proteína [5]. Así mismo, P. ostreatus puede presentar variabilidad de porcentajes de proteína al cultivarse en otros sustratos como: la paja de trigo $25,6 \%$, eucalipto $25,4 \%$, paja de eucalipto $24,4 \%$ y en álamo 22,9\% [6]. Para el porcentaje de proteína, el T2 fue ligeramente superior al T1, 
lo que puede ser producto de la expresividad genética del hongo con respecto de la naturaleza del sustrato (aserrín) que al ser mucho más crudo puede contener mayores porcentajes de lignina y celulosa [31, 32] generándose mayor cantidad de proteínas como lacasa [33].

Varias muestras presentaron valores de proteína fuera de los rangos medios obtenidos, los mismos que fueron descartados como errores por una triple validación de la prueba con dichas muestras. Estos valores, probablemente se deban a posibles adaptaciones genéticas de cada muestra individual incrementando la expresividad génica y proteínica [34].

Con este estudio y aplicación del cultivo Pleurotus ostreatus usando recursos accesibles y económicos, sin controles automatizados de variables como la temperatura y humedad se ha logrado obtener un producto con un excelente porcentaje de proteína, contribuyendo con los objetivos de desarrollo sostenible (ODS), como cambiar el mundo con el fin de la pobreza o hambre cero (ODS 1 y 2), además de ayudar a poblaciones vulnerables económicamente del sector, y aportando al conocimiento de cultivo y valorización de los hongos.

\section{AGRADECIMIENTOS}

Agradecemos al financiamiento de reactivos y disponibilidad de laboratorios de Cultivo y Conservación de Microorganismos y Laboratorio de Química de la Universidad Técnica Particular de Loja UTPL.

\section{CONTRIBUCIONES DE LOS AUTORES}

Darío Cruz y Ruby Ojeda concibieron la investigación; Darío Cruz y Ruby Ojeda desarrollaron la metodología y realizaron el trabajo de campo; Diego Maza y Ruby Ojeda desarrollaron metodología de laboratorio; Ángel Benítez diseñó el modelo y corrió los análisis; Darío Cruz y Ángel Benítez redactaron el manuscrito; Ruby Ojeda, Daniel Capa y Diego Maza revisaron críticamente el contenido intelectual del manuscrito; Darío Cruz tomó la fotografía de la figuras 1; Ángel Benítez aporto con las figuras 2, 3 y 4. 


\section{REFERENCIAS}

[1] Pavlik, M., Hraško, M., \& Pavlíková, A. (2008). Oyster mushroom Pleurotus ostreatus (Jacq.) P.Kumm. - its cultivation and utilization in the Slovak forestry. In Mushroom biology and mushroom products. Proceedings of the Sixth International Conference on Mushroom Biology and Mushroom Products, Bonn, Germany, 29 September-3 0ctober, 2008 (pp. 246-254). GAMU GmbH, Institut für Pilzforschung.

[2] Moore, D. (2005) Principles of mushroom developmental biology. International Journal of Medicinal Mushrooms, $7: 1-2$

[3] Ramón, P., \& Ramón, D. (2012). Análisis de la capacidad degradativa de residuos lignocelulósicos utilizando el hongo Pleurotus ostreatus var. Florida (Universidad Politecnica Salesiana Sede Cuenca). Retrieved from https://dspace. ups.edu.ec/bitstream/123456789/2811/6/UPS-CT002462.pdf

[4] Nieto, I. J., \& Chegwin, C. (2010). Influencia del sustrato utilizado para el crecimiento de hongos comestibles sobre sus características nutraceúticas. Revista Colombiana de Biotecnología, 12(1), 169-179.

[5] Motato, K., Mejía, A., \& León, Á. (2006). Evaluación de los residuos agroindustriales de plátano (Musa paradisíaca) y aserrín de abarco (Cariniana piriformes) como sustratos para el cultivo del hongo Pleurotus djamor. Vitae, 13(1), 24-29.

[6] Varnero, M. T., Quiroz, M.S., \&Álvarez, C. H. (2010). Utilización de residuos forestales lignocelulósicos para producción del hongo ostra (Pleurotus ostreatus). Informacion Tecnologica, 21(2), 13-20. https://doi.org/10.16124154i09.

[7] Pineda, I. J., Ramos-Sánchez, L. B., \& Soto-Arroyave, C. P. (2014). Producción de Pleurotus ostreatus por fermentación en estado sólido: una revisión ICIDCA. Sobre los Derivados de la Caña de Azúcar,48(2), 13-23.

[8] Pineda, I. J., Soto-Arroyave, C., Guzmán-Torres, R., Santiago-Vispo, N., Huaca-Pinchao, J., Duarte-Trujillo, S., \& Pineda-Soto, A. (2016). Producción de hongo ostra (Pleurotus spp.) en bagazo de caña ICIDCA. Sobre los Derivados de la Caña de Azúcar, 50(1). 50-54

[9] Santillán, T. M.M., \& Morocho N. V. E. (2018) Evaluación de sustratos lignocelulósicos para la producción del hongo ostra (Pleurotus ostreatus), en la parroquia Tarqui. Faculdad de Ciencias de la Tierra; Carrera de Ingenieria Agropecuaria; Universidad Estatal Amazónica.

[10] Patil, S., Ahmed, S., Telang, S., \& Baig, M. M. V. (2010). The nutritional value of Pleurotus ostreatus (JACQ.:FR.) Kumm cultivated on different lignocellulosic agrowastes. Innovative Romanian food biotechnology, 7.66-76.

[11] Barros, L., Cruz, T., Baptista, P., Estevinho, L. M., \& Ferreira, I. C. (2008). Wild and commercial mushrooms as source of nutrients and nutraceuticals. Food and Chemical Toxicology, 46(8), 2742-2747. https://doi.org/10.1016/j. fct.2008.04.030.

[12] Cohen, R., Persky, L, \& HadarY. (2002) Biotechnological applications and potential of wood-degrading mushrooms of the genus Pleurotus Appl Microbiol Biotechnol 58:582-594 D0I 10.1007/s00253-002-0930-y.

[13] Svanberg, D., \& Lindh, H. (2019). Mushroom hunting and consumption in twenty-first century post-industrial Sweden; Journal of Ethnobiology and Ethnomedicine 15(1),42 https://doi.org/10.1186/s13002-019-0318-z.

[14] Gamboa-Trujillo, P., Wartchow, F., Cerón-Martinez, C., Andi, D., Uwinjin, P., Grefa, G., \& Piyaguaje, N. (2019). Edible Mushrooms of Ecuador: consumption, myths and implications for conservation. Ethnobotany Research and Applications, 18, 1-15.

[15] Majesty, D., ljeoma, E., Winner, K., \& Prince, 0. (2019). Nutritional, anti-nutritional and biochemical studies on the oyster mushroom, Pleurotus ostreatus. EC Nutrition, 14(1), 36-59. "Nutritional, Anti-nutritional and Biochemical Studies on the Oyster Mushroom, Pleurotus ostreatus." EC Nutrition 14.1: 36-59.

[16] Kakon, A. J., Choudhury, Md B. K., \& Saha, S. (2012). Mushroom is an ideal foodsupplement. Journal of Dhaka National Medical College \& Hospital, 18(1),58-62.

[17] Aghajani, H., Bari, E., Bahmani, M., Humar, M., Ghanbary, M.A. T., Nicholas, D.D., \& Zahedian, E. (2018). Influence of relative humidity and temperature on cultivation of Pleurotus species. Maderas Ciencia Tecnología. 20(4), 571-578

[18] López-Rodriguez, C.; Hernández-Corredor, R.; Suárez-Franco, C. \& Borrero, M. (2008). Evaluación del crecimiento y producción de Pleurotus ostreatus sobre diferentes residuos agroindustriales del departamento de Cundinamarca. Universitas Scientiarum, 13(2), 128-137. 
[19] Aguinaga, P. (2012). Evaluación de cuatro sustratos para la producción del hongo ostra (Pleurotus ostreatus) en tres ciclos de producción en la zona de Tambillo, provincia de Pichincha (Tesis de pregrado). Escuela Politécnica Nacional, Quito.

[20] Gaitán-Hernández, R., Salmones, D., Pérez-Merlo, R., \& Mata, G. (2006). Manual práctico del cultivo de setas: aislamiento, siembra y producción. Instituto de Ecología, AC, Xalapa, México.

[21] Vega, A., \& Franco, H. (2013). Productividad y calidad de los cuerpos fructíferos de los hongos comestibles Pleurotus pulmonarius RN2 y P. djamor RN81 y RN82 cultivados sobre sustratos lignocelulósicos. Información Tecnológica, 24(1), 69-78. https://doi.org/10.4067/S0718-07642013000100009

[22] Becker, J. (2012). Plasmons as sensors. Tesis Springer. https://doi.org/10.1007/978-3-642-31241-0.

[23] Bermúdez, R., García, N., \& Mourlot, A. (2007). Fermentación sólida para la producción de Pleurotus sp. sobre mezclas de pulpa de café y viruta de cedro. Tecnología Química, XXVII(2), 55-62.

[24] Guzmán, D., Herrera, J., Fuentes, E., \& Hernández, A. (2019). Evaluación del crecimiento y producción del hongo ostra Pleurotus ostreatus bajo condiciones artesanales utilizando restos de cosecha en el municipio de Camotán, Chiquimula, Guatemala. Tikalia, XXXVII, No 1, 23-40.

[25] Valencia de Ita, M. Á., Castañeda, M. D., Huerta, M., \& Romero, 0. (2019). Carrizo silvestre (Arundo donax) como sustrato alternativo en la producción de Pleurotus ostreatus. Scientia Fungorum, 48, 15-22. https://doi.org/10.33885/ sf.2018.48.1231.

[26] Rios, P., Hoyos, J., \& Mosquera, S. (2010). Evaluación de los parámetros productivos de la semilla de Pleurotus ostreatus propagada en diferentes medios de cultivo. Facultad de Ciencias Agropecuarias, 8 (2), 87-93.

[27] Silva, R., Fritz, C., Cubillos, J., \& Díaz, M. (2010). Manual para la producción de hongos comestibles (Shiitake). Santiago, Chile: Proyecto CONAMA-FPA.

[28] Donado, T. (2014). Evaluación de tres sustratos para la producción de hongo ostra (Pleurotus ostreatus); Moyuta, Jutiapa (Universidad Rafael Landivar).

[29] Caicedo, P. (2013). Utilización de residuos orgánicos lignocelulósico provenientes de actividades industriales para la producción de hongos comestibles Pleurotus spp. en el distrito especial de Buenaventura, Colombia.

[30] Bonatti, M., Karnopp, P., Soares H. M., \& Furlan S. A. (2004). Evaluation of Pleurotus ostreatus and Pleurotus sajor-caju nutritional characteristics when cultivated in different lignocellulosic wastes. Food Chemistry 88(3):425-428.

[31] Sánchez, C. (2010). Cultivation of Pleurotus ostreatus and other edible mushrooms. Applied microbiology and biotechnology, 85(5), 1321-1337. doi: 10.1007/s00253-009-2343-7.

[32] Sánchez, J. E., \& Royse, D.J. (2001). La biología y el cultivo de Pleurotus spp. El colegio de la frontera sur. México.

[33] Janusz G, Kucharzyk, K.H., Pawlik, A., Staszzzak, M., \& Paszzzynski, A. J. (2013) Fungal laccase, manganese peroxidase and lignin peroxidase: gene expression and regulation. Enzyme and Microbial Technology; 52(1):1-12.

[34] Amore, A., Giacobbe, S., \& Faraco, V. (2013) Regulation of cellulase and hemicellulase gene expression in fungi. Current genomics, 14(4), 230-249. 